\title{
A FORENSIC-BASED STUDY ON LOW ANGLED AK RIFLE BULLET ENTRY WOUNDS USING A PORCINE MODEL
}

Wounds from gunshots occur when a projectile or pellets fired from any given firearm enters a human or animal body and depend on many factors, including the projectile itself, target surface and direction and rate of transmission of energy (Eason et al. (1975). Various types of firearms and bullets may produce wounds that have different characteristics reflective of the weapon system inflicting the injury (Frost R.E and Denton J.S, 2015), assisting forensic pathologists to interpret gunshot wounds and answer the related medico-legal questions. Additionally, pathological findings from gunshot wounds also assist forensic investigators in event reconstruction (Haags, 2011).

Out of the two main types of gunshot wounds; entry and exit, an entry wound normally carries more macroscopic and microscopic evidence than found in exit wounds. For example, entry wounds may show an abrasion collar, burn marks, large amounts of trace evidences from the bullet and gun such as gunshot residues, soot, powder tattooing, a grease ring (bullet wipe) and muzzle imprints, making them a richer source of evidence than exit wounds.

Entry wound characteristics for contact, near contact, intermediate and distant wounds have been discussed in existing literature adequately with reference to rifled and smooth bore guns (i.e. Di Maio (1999), Peonim et al. (2016), Frost R.E and Denton J.S (2015), Wong et al. (2013), Molina and Dimaio (2013), Molina et al. (2007), Apfelbaum et al. (1998), Randall and Jaqua (1990), Knoop et al. (2010)). Furthermore, research studies on gunshot wounds such as macroscopic and microscopic characteristics of entry wounds produced by ricocheting bullets (Yong (2017) destabilised projectiles (DiMaio (1999) and histological analysis of dermal gunshot wounds (Adelson (1961) have also been reported for different ammunition types and ranges. However, there is limited scientific work that specifically focuses on the entry wound characteristics of low-angled rifle and hand gun projectiles. General wound features of low-angled bullet impact on skin such as comet-tailed features (Knoop et al. (2016)) and an increasingly oval shape with increasing impact angle (Eason et al. 1975) are explained in many publications, but there seem to be a lack of wider, multiple variable studies in this area that consider both macroscopic and microscopic wound characteristics in human, porcine or any other tissue substitute. During a low angle impact, it is understood that different types of bullet design interact with the skin over a 
greater surface area and with more interaction time than a right-angled entry. As a result, such interactions have the potential to produce forensically-important macroscopic and microscopic wound characteristics unique to each condition.

This study was devoted to understanding the entry wound characteristics of low-angled rifle bullets on porcine skin. Porcine skin was used for the study as it has been proved as a suitable mimic for human skin in medical and forensic research due to similar histological and immunological properties (Avon and Wood (2005)). An AK-type rifle was used for the experiment as bullets used with such rifles; $7,62 \mathrm{mmx} 39 \mathrm{~mm}$ are reported with great regularity in shooting incidents around the world and are arguably one of the most commonly-used bullet type encountered by forensic pathologists and shooting investigators.

\section{MATERIAL AND METHODS}

\section{Experimental Arrangement}

A mounted AK-type rifle (Chinese made Type 56 MK-2 rifle with foldable iron butt) was fired into porcine skin samples placed on a target tray at different 'low angles'. The ammunition used was Chinese made 7,62x39mm BALL with full metal jacket (FMJ) and lead (steel) core.

The rifle was firmly fixed on to a gun mounting platform to ensure there were no changes to the gun barrel or gun position during the firing of each shot. The gun and the barrel were kept exactly horizontal to the ground and the level was regularly checked using a bubble level. The accuracy of the level was re-confirmed before firing each shot. The barrel height (height from the floor level to the muzzle end) was $1.6 \mathrm{~m}$.

A target holder specially designed to hold porcine skin samples at different angles was placed 10 metres away from the muzzle end of the mounted rifle. The base of the target holder, from which the angles of the target tray were measured was kept exactly parallel to the ground (and axis of the bore) and checked using an inclinometer for accuracy prior to the discharge of each shot. The inclinometer precision was plus or minus 0.10 degrees. The impact point of the bullet on the porcine skins were set to align with the barrel height; $1.6 \mathrm{~m}$. The experimental setup including the gun mount and target holder is shown in Fig 1. 


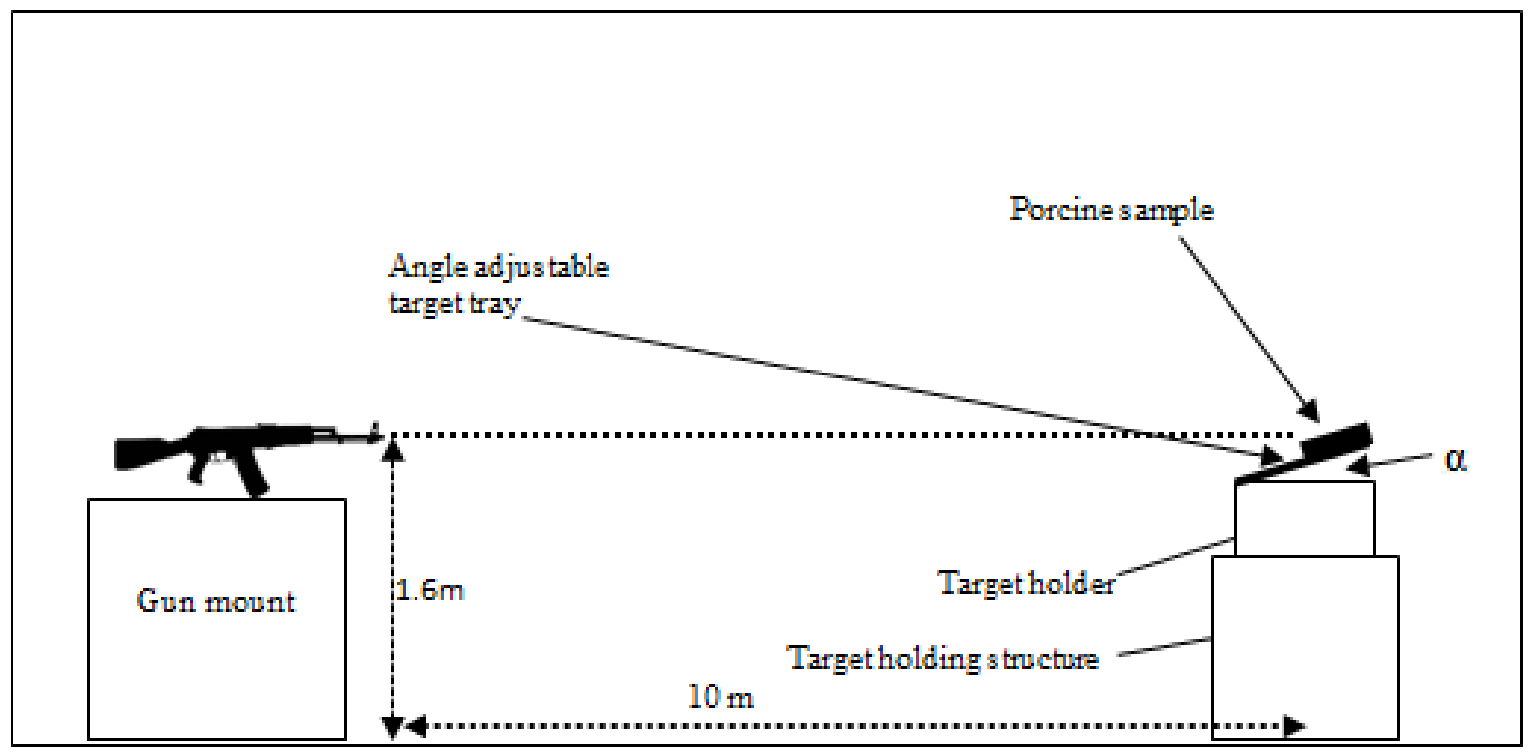

Figure 1 : The Experiment set up

After setting up the experimental apparatus, 5 shots were fired to a flat jack wood piece placed on the sample holder at 90-degrees to the bullet's trajectory. This was done to test the experimental apparatus. The 5 shots produced clean bullet holes with no indication of yawing on the jack wood sample. This confirmed that the bullets were striking the target with their nose pointed forward as a result of gyroscopically stabilised flight at the 10-metre range used.

\section{Porcine samples}

Fresh porcine skin samples for the experiment were collected from an already dead animal and not harmed for research purposes. The samples were received five hours after the animal was killed and then stored in a freezer for 24 hours. The skin samples were cut in to eight $(20 \mathrm{x} 20$ $\mathrm{cm})$ pieces, each being approximately $5 \mathrm{~cm}$-thick, including subcutaneous tissue, before then being refrigerated. All porcine samples were cut from flat skin surface areas of animals and maximum effort was taken to cut samples with right angled edges. Before starting the experiment, all the samples were taken from the refrigerator and kept outside for three hours until all ice melted and they reached ambient temperature. Porcine skin samples were fixed to a jack wood board with natural tension and four pins were used to fix the skin samples to the board. Once the samples were prepared, the jack wood tray with the porcine sample was placed on the angle adjustable tray of the target holder at different angles to the gun's bore axis. 
A pre-test firing session was conducted to check the experimental set up and to decide the range of low angles for data collection for the experiment. Based on the observations of the pre-test firing, data collection commenced from a 17-degree incident angle, where the entry wounds started to showcase an expanding shape (All other wounds above 17 degrees displayed a round shaped entry as seen in a right-angled shot). Angles of incidence were then continuously decreased and stopped when the bullet started to make a long-wound track on the surface of the porcine sample passing along its full $20 \mathrm{~cm}$ length. This resulted in 7 angles being interrogated between 17 and 6 degrees. 10 shots were repeated at every incident angle and 5 to 6 shots collected to one porcine sample by moving the target holder left to right without changing the original distance from the muzzle end and other alignments. This was done as the length of a single porcine sample was not sufficient to collect all 10 shots. As such, 10 entry wound samples from each angle were collected for analysis. All entry wounds were photographed and numbered before being removed from the target tray. Once removed, the measurements of the wounds were taken using a digital calliper and all external wound characteristics were recorded.

After the measurement taking and recording all external characteristics with photographs, porcine samples with entry wounds were separately stored in glass containers with formalin (mixture) and sent to the laboratory for preparation for histology. One wound sample out of 10 sample of each angle fired were selected randomly for histology and samples were taken from begin, middle and end of every wound for preparation of slides. A pathologist was consulted to interpret the histology results.

\section{RESULTS AND DISCUSSION}

\section{External Wound Characteristics}

At the lowest angles (6 and 7 degrees), the fired bullet glanced across the tissues producing an injury similar to a laceration or graze wound, whereas at the highest incident angles (15 and 17 degrees), the bullets made a clear entry hole similar to a direct shot. The wounds were carefully analysed and all were found to have bullet wipe present, which also provided a clue as to the direction of the bullet's approach. Photos of the entry wounds from 6 to 17 degrees are presented in Figure 2. 

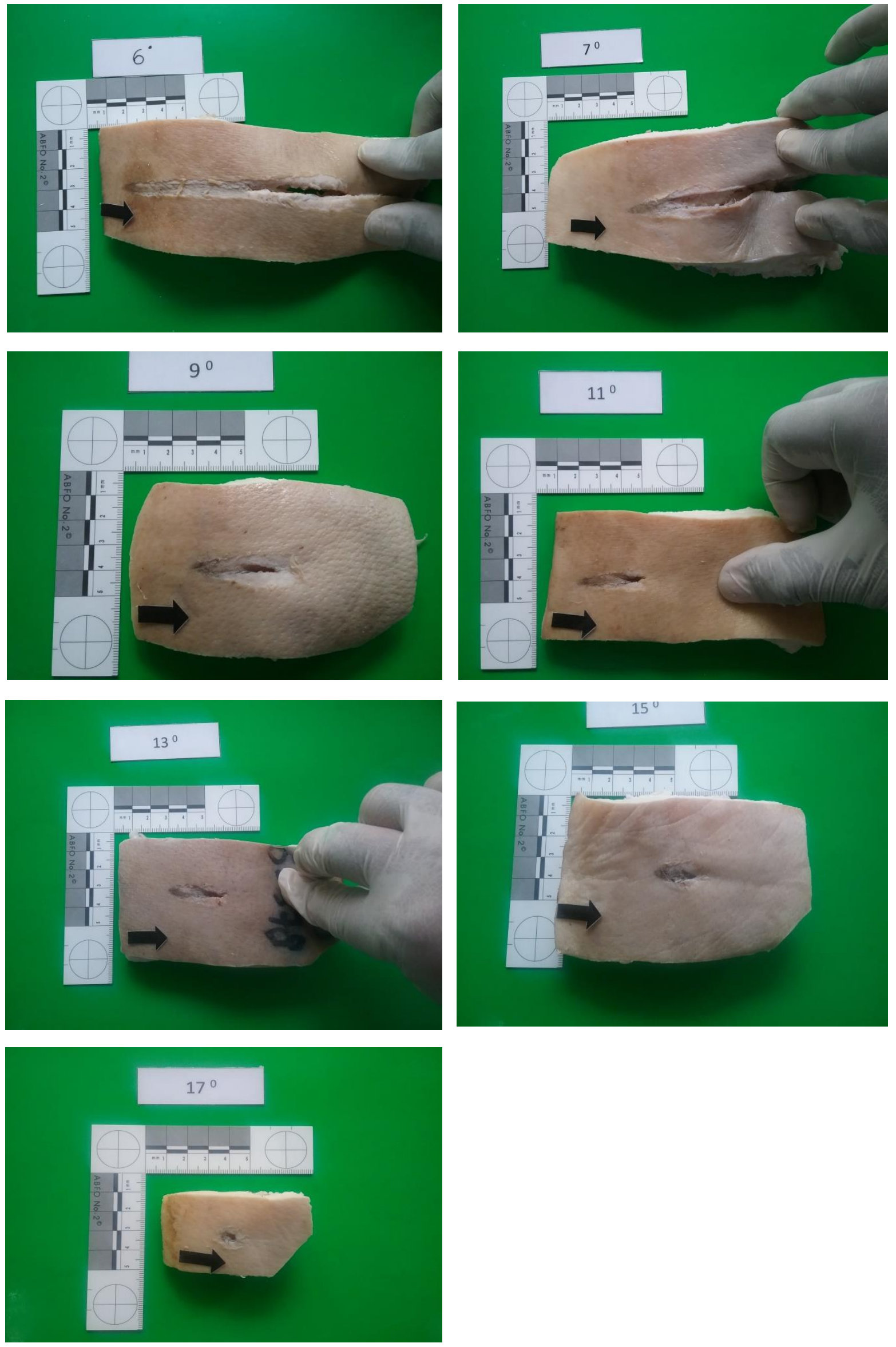

Figure 2 : General wound characteristics from 6 to 17-degrees 
The following are the observations and interpretations with regards to the macroscopic entry wound characteristics observed for the wound samples:

a. Absence of the typical "abrasion ring/collar" - Instead of a standard abrasion ring or collar, which is a common observation for a bullet entry wound, a different abrasion ring feature was observed in all the wounds irrespective of the angle of incidence. In all entry wounds, specifically, layers of the top epidermis were seen loosened, damaged and receded, producing a visible white patch around the wounds with damaged outer margins. (Fig 3).
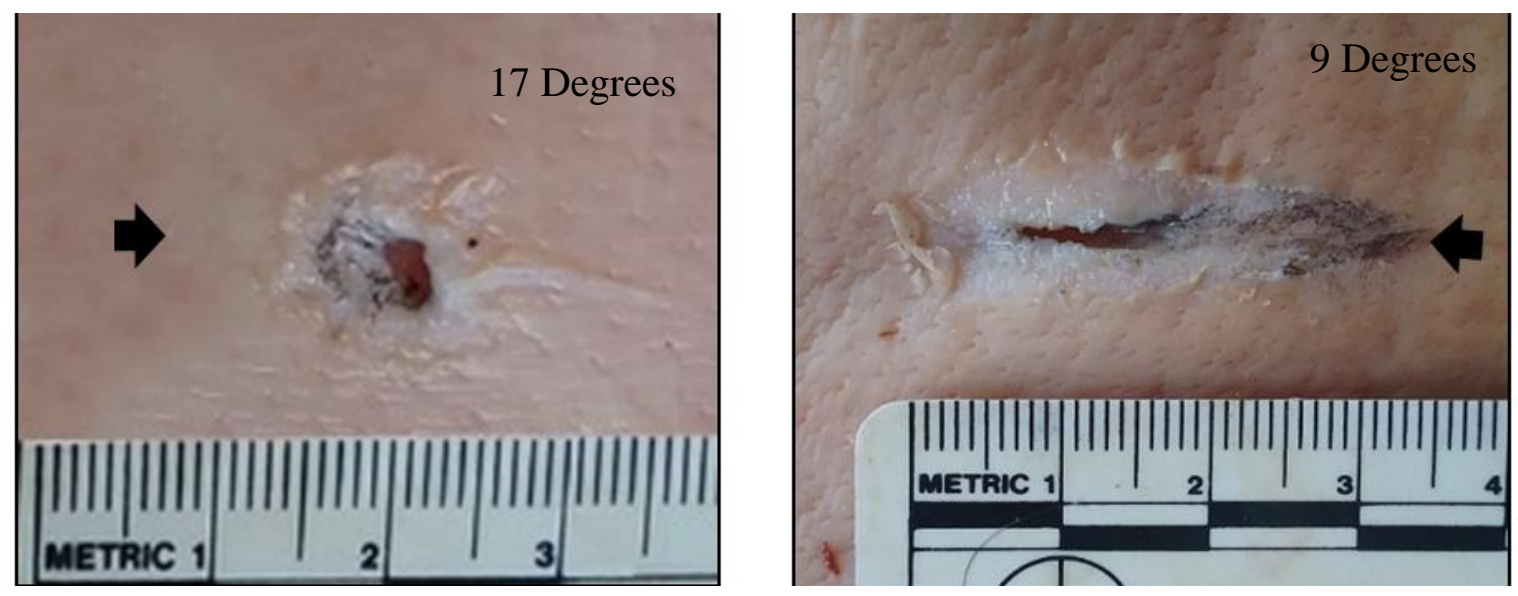

Figure 3 : A special abrasion ring feature with loosened and damaged skin around the entry holes were observed in all entry wounds. An example of this feature in 17 and 9 degrees are illustrated above. The arrow shows the direction of bullet travel.

According to DiMaio (1999), wounds from high velocity centre-fire rifle bullets sometimes do not produce an abrasion ring. He also explains that sometimes entry wounds from rifles have small splits or tears radiating outwards from the edges of the perforation. The features called "micro-tears" present around the complete or partial circumference of the wound. The same entry wound characteristics has also been reported by Peonim et al. (2016) with M-16 high velocity rifle bullets. However, the abrasion ring feature observed in this work was different to those shown by DiMaio and 
Peonim and some of the other available descriptions and pictorial illustrations on the "abrasion patch" or "abrasion collar" in the existing literature (Knoop, K.J et al. (2010), Frost R.E and Denton J.S (2015) and Mussen, D (2020). The destruction and micro-tears on the outer margin of the abraded area for all wound samples here are the main feature highlights, which differ with the standard abrasion patch/collar explained and illustrated in the existing literature. A comparison highlighting the difference between an ordinary abrasion ring/micro-tear feature for gunshot wounds highlighted in the existing literature and a newly observed abrasion ring feature at 17-degrees is shown in Fig. 4 for comparison.
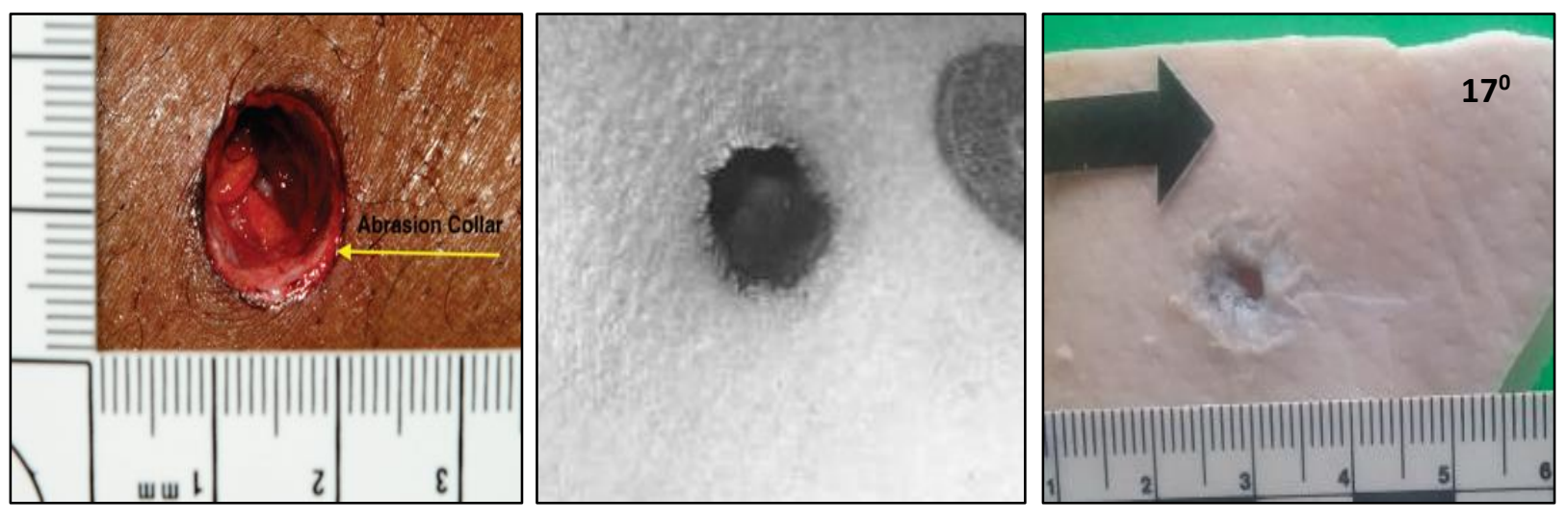

Figure 4 : Difference of an ordinary abrasion ring of a gunshot wound (Left, Knoop et al. (2010)), micro tears (middle, Fitchett (2020)) and the newly observed abrasion ring feature (Right) at a 17-degree incident angle with no clear outline and micro tearing around the outer margins of the abraded area.

During low angle impact with the skin, bullets transfer their energy to the epithelium over an extended interaction time and a larger area than a direct hit. This angular interaction and forward thrust of the bullet with the transmitted energy over a large area may cause the epithelium around entry wounds to damage differently, producing an abrasive patch with damaged outer margins instead of "a standard abrasion ring" or "micro tears". This also may be due to the high velocity of centre fire AK bullets with a copper jacket as highlighted by Di Maio (1999), other factor relating to the bullet such as the shape (Pricher et al. (2016) or more likely as a result of the differing elastic properties of the 
layers of the skin affecting the overall behaviour of the tissue system. This will therefore influence the overall energy transfer during the projectile-tissue interaction.

Alternatively, the observations could be due to post-mortem changes in the skin, with the epidermis beginning to separate from under lying dermis resulting from the weakening of the skin layer interfaces (Cohut, 2018) and a result of the moist conditions experienced during the refrigeration of skin samples, also could have been the reasons for this unique feature. Consequently, these features may have been different if the test was performed with the animal in deep surgical anaesthesia as reported in researches conducted by Easen et al. (1975) and Avon and Wood (2005), using live pigs.

b. Bullet wipe - Bullet wipe is residue and/or lead from the surface of the bullet that is transferred to clothing or skin and can be seen at any range of fire (Knoop et al. 2010). This was seen in each of the wound samples collected irrespective of the angle of incidence (Figures 3, 4, 5 and 6). However, it was understood that the bullet wipe features maybe observed with varying density based on the number of shots fired prior to the wound inflicted as the amount of residue and lead deposit in the gun barrel will raise with increased numbers of shots and will also differ based on gun and ammunition types. In the shots performed here, bullet wipe was mostly seen on the part of the wound where initial contact was made between the bullet and skin and the density of the wipe decreased in the direction of travel. This provides a strong indicator of directionality for bullet wipe's use as informative forensic evidence in shooting cases.

c. Comet tail -_Elongated abrasion collars from projectiles which enter the skin at low impact angles may produce a collar with a "comet tail" (Knoop et al. (2010). The comet tail is produced due to the low approaching angle of the bullet relative to the skin and subsequent lifting of the immediate front section of the skin due to the pressure imparted by the nose when it comes into first contact with the skin. The same features were observed in all incident angles except 17-degree wounds (Figure 5). However, as explained in sub-paragraph "a" above, the standard abrasion collar described in the existing literature was not observed here too. 


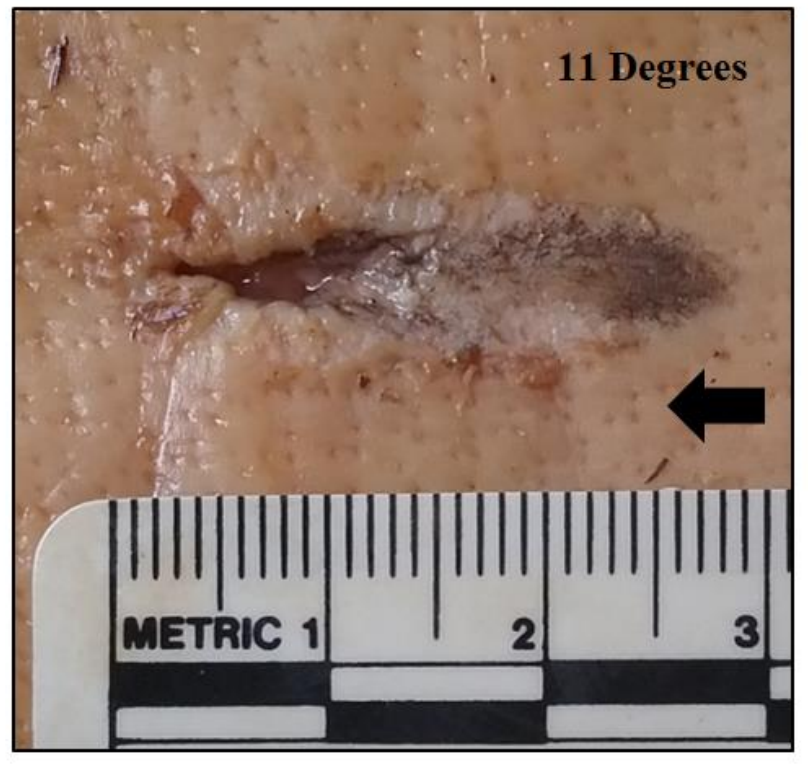

Figure 5: A clear comet-tailed effect of an 11-degree entrance wound. Black arrow indicates the direction of approach of the bullet.

d. Approximate lengths of the entry wounds - As the diameter of the bullet hole was difficult to measure, due to the low-angled impacts causing elliptical wounds throughout, the approximate lengths of the wounds were measured from the beginning of the wound to the end, including the bullet wipe. The measurements were taken using a digital calliper and an example of a typical wound length is illustrated in Figure 6. 


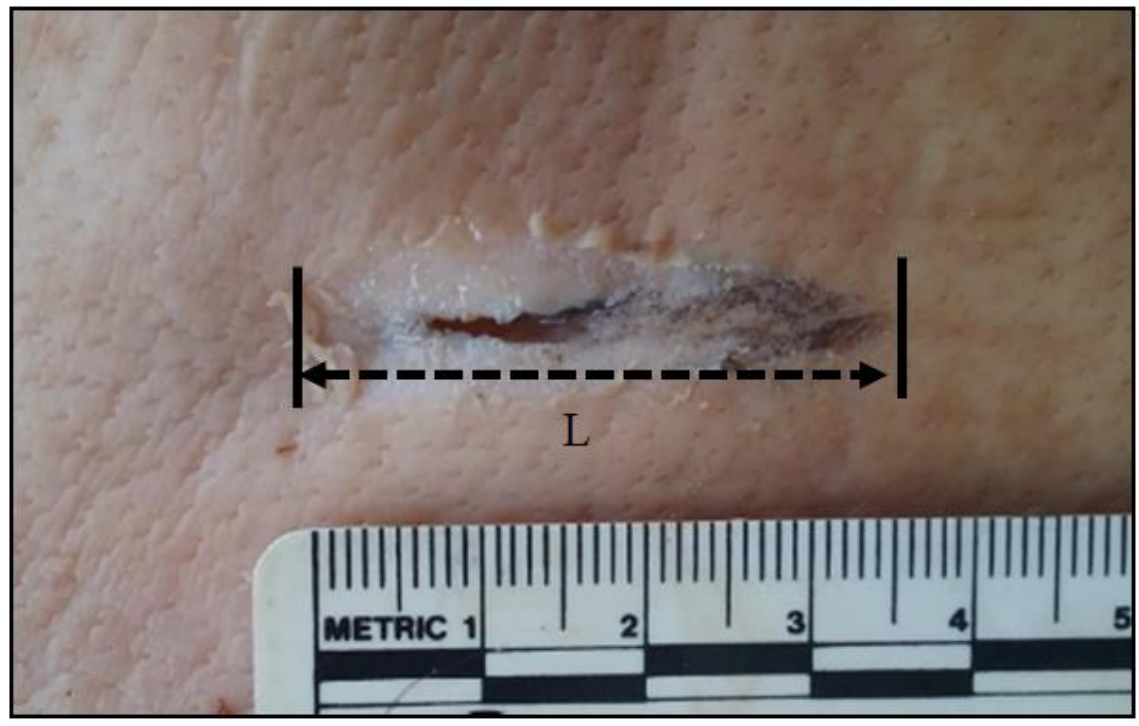

Figure 6 : An example of measuring the approximate length of a wound (L) produced at 9 degrees

All measured wound lengths demonstrated a strong relationship with their respective angles of incidence for the bullets fired. This relationship is statistically shown in Figure 7 as being linear in nature with a negative gradient. Although it has been explained in the literature in general about the increasing oval shape of an angled shot, its numeric relationship with human or porcine skins has not been highlighted in the forensic ballistic or wound ballistic related literature before. The numerical relationship revealed that the lengths of the entrance wound in this experiment can be considered as vital supporting information for forensic pathologists to understand an AK bullet's approaching angle to a victim's body and its subsequent projectile path determination. However, it should be noted that findings with regards to the lengths of the entry wound of this research can be referred only to AK bullet entries on flat areas of the body. 

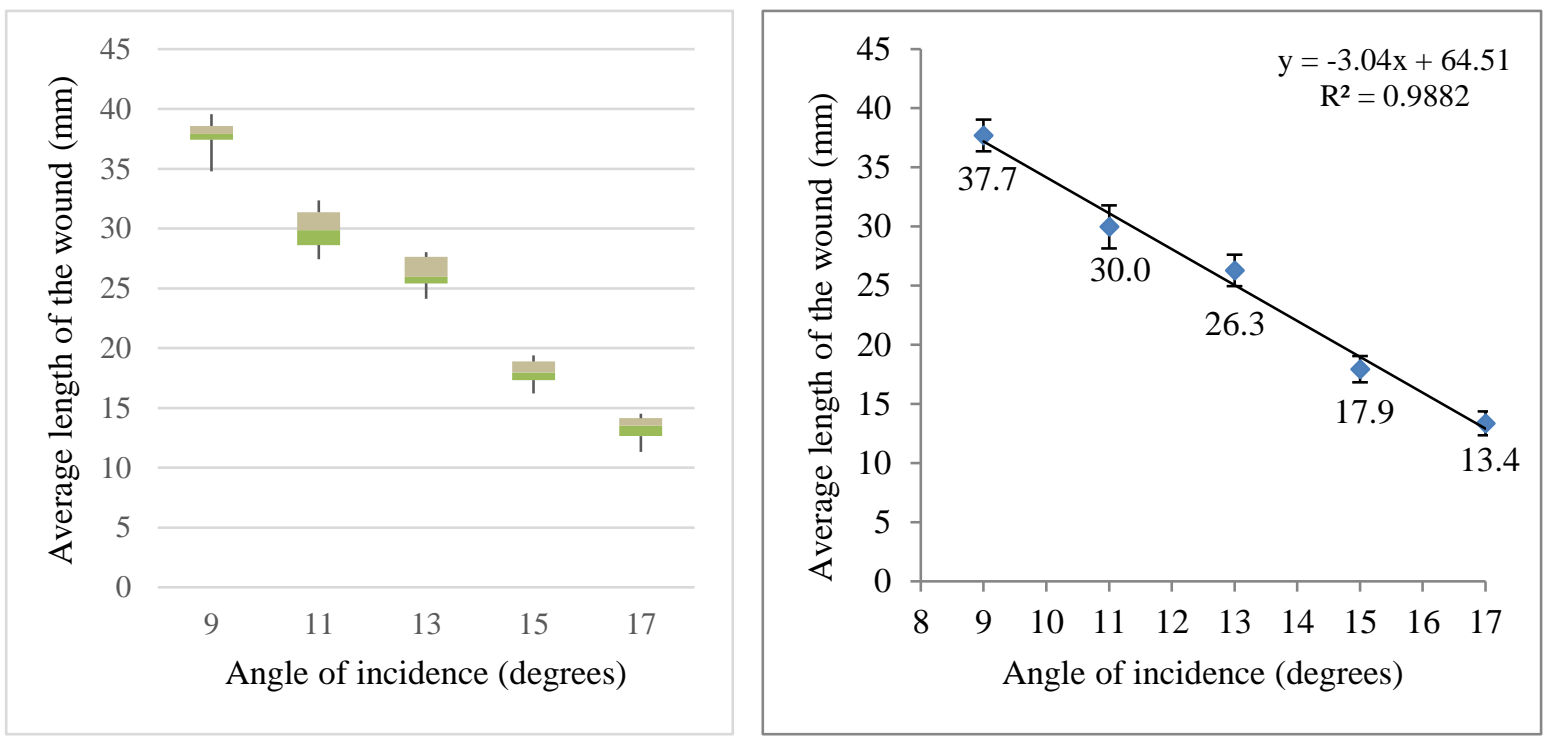

Figure 7: A statistical representation of the data and the relationship between the angle of incidence of the AK bullets used and the length of the wound. The length of the wound for 6 and 7-degrees were not included since the length of the wound continued beyond the size of the sample.

\section{HISTOLOGY}

Histology results of firearm wounds of this study did not showcase much important or meaningful pathological information in relation to the angles of incidences. However, general histological features of the wound samples were confirmed to the histology of gunshot wounds reported in previous studies (Adelson, L 1961). The histology features observed in randomly selected wound samples from each angle of approach is presented below at figure 8 . 

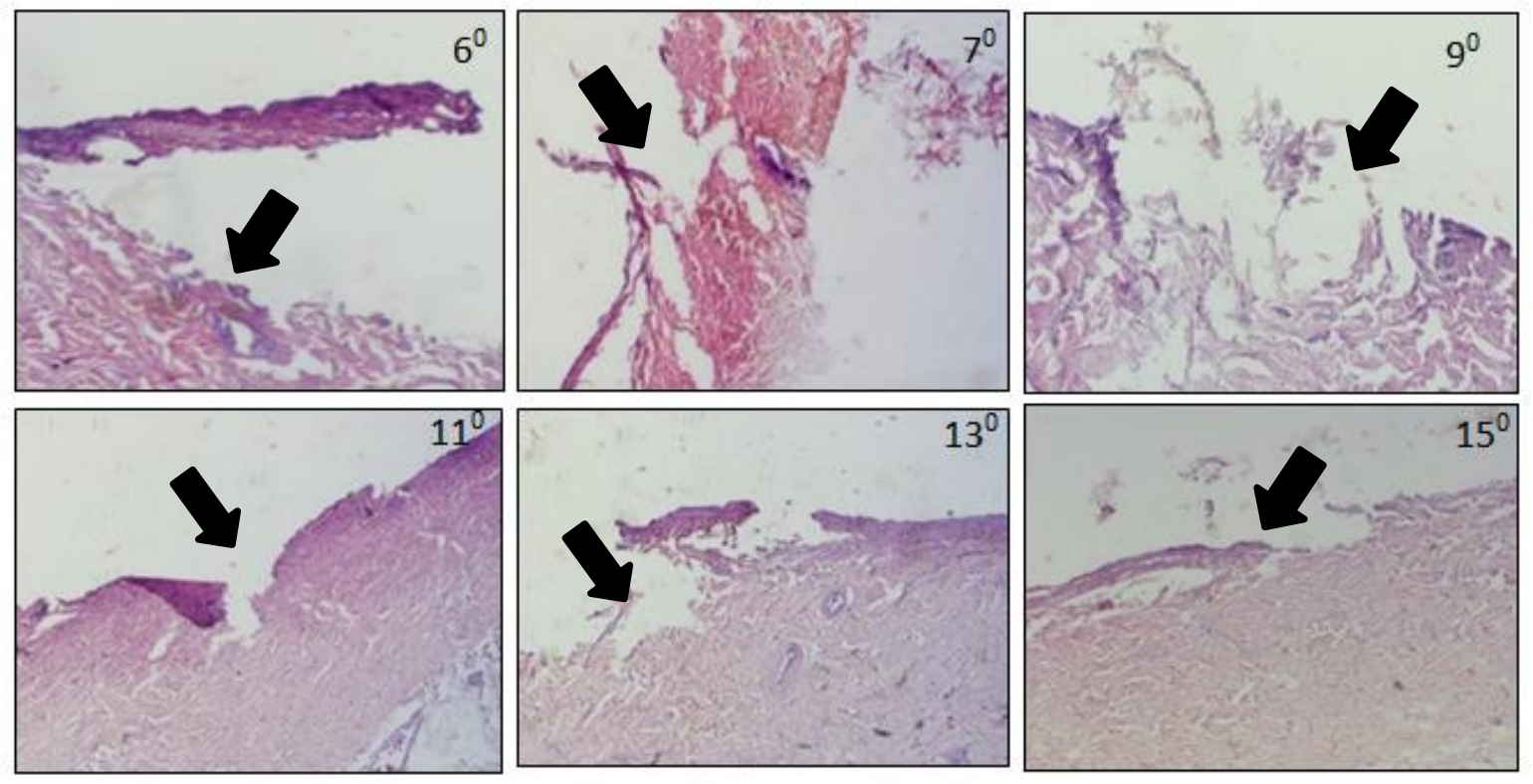

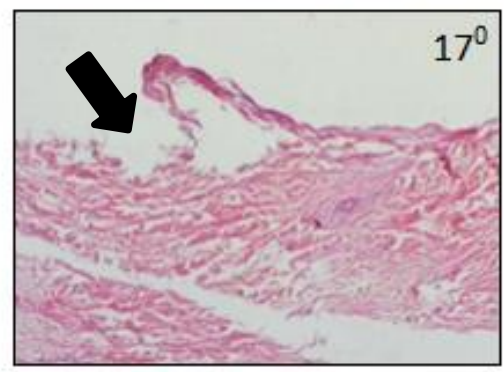

Figure 8 : Histology features observed in randomly selected wound samples from each angle of approach. Damage to the epithelium is highlighted with arrows.

Epithelial damage caused by mechanical trauma due to the low angle impact of the bullet was significantly observed in all samples irrespective of angles of approaches. Damages to the epidermal architecture due to the mechanical trauma, cellular disruptions, distortions and total epithelial destruction were seen in histology samples, supporting the findings of Adelson (1961). However, this was observed haphazardly; not showing any pattern or trend in relation to bullet's incident angle or the location of the wound. Additionally, no post-mortem changes were observed in the samples. 


\section{Conclusion}

This study aimed to better understand the low angle entry wound characteristics of AK bullets on porcine skin. The common abrasion ring feature reported in the literature was not observed in any of the wound samples. Instead of a standard abrasion ring being present, a unique abrasion ring feature around the entry holes was observed where the layers of the epidermis were seen loosened and damaged producing a visible patch with damaged outer margins around the entry wounds. To the authors' knowledge this new feature has not been previously reported in the existing literature, either visually or in discussion. Thus, it may be worth extending this study in the future to consider whether this feature is unique to low angled AK bullet entry wounds, whether it occurs more widely amongst other ammunition types or if this was a substrate-related characteristic resulting from the condition or properties of the skin samples used for this study.

A previously unreported relationship was observed between the angles of incidence of the AK bullets and the lengths of the wounds created and must be considered as an important finding of this research from both pathological and shooting investigation points of view. This has the potential to be a useful investigative tool alongside other available wound-related evidence. From the pathologist's point of view, the findings may help to understand the angle at which the bullet approached a victim's body from, during the gross examination of entry wounds and subsequent identification of the wound track. In the investigative aspect, wound lengths observed from different incident angles in this experiment proved to be a viable reference in identifying the bullet's approximate approaching angle. This is currently only suitable for where a "lowangled" (below 17 degrees) AK bullet entry is observed on a flat area of a victim's body and the victim's approximate position prior to impact by the causative bullet is known (i.e. sleeping, sitting etc). However, the natural unevenness and variability of the human skin and the effects of underlying bones must be considered when applying these numerical values to understand the bullet's approaching angles. Overall, it is believed that this work opens up new avenues of research in this field. 


\section{$\underline{\text { Reference }}$}

Adelson S. A microscopic study of dermal gunshot wounds. Am J Clin Pathol.1961; 35-5: 393402.

Avon S.L, Wood R.E (2005) Porcine skin as an in-vivo model for ageing of human bite marks. J FORENSIC ODONTOSTOMATOL. 2005; 23-2: 1. Available at:

https://pdfs.semanticscholar.org/bdde/005d8cb726b1c863a72cee0fb1b633166079.pdf?_ga=2.15 $3037689.1704580405 .1573753575-9796830.1550077857$

Apfelbaum J.D, Shockey, L.W, Moore, E.E (1998) Entrance and Exit gunshot wounds, The Jpurnl of Emergency Medicine. DOI: https://doi.org/10.1016/S0736-4679(98)00075-4

Bev Fitchett (2020) https://www.bevfitchett.us/gunshot-wounds/entrance-versus-exit-woundsentrance-wounds.html

Cohut, M 'What happens to the body after death?' Medical News Today, 11 May 2018. Available at: https://www.medicalnewstoday.com/articles/321792.php\#1

DiMaio VJM. Gunshot wounds, Practical Aspect of Firearms, Ballistics and Forensic Techniques. $2^{\text {nd }}$ edn. New York. CRC Press. 1999. 
Eason R.L, Pryor W.H, Adams J.F (1975) Wound studies in porcine skin, muscle and liver as related to variation of velocities of spherical missile, Naval Medical field Research Laboratory Test Report. Available at: https://apps.dtic.mil/dtic/tr/fulltext/u2/a004636.pdf. Accessed September 13, 2019

Frost R.E and Denton J.S (2015) Forensic Pathology of Firearm Wounds. Available at: https://emedicine.medscape.com/article/1975428-overview. Accessed October 2019

Haag MG, Haag MC. Shooting Incident Reconstruction. First edn. Elsevier Inc. 2011

Knoop, K.J, Stack, L.B., Storrow, A.B., Truman, R.A (2010) Atlas of emergency Medicine $3^{\text {rd }}$ edn. Mc-Graw Hill Companies

Knoop, K.J, Stack, L.B, Storrow, A.B and Thruman, R.J (2016) Atlas of emergency Medicine, Forensic Pathology (2019) https://www.barnardhealth.us/forensic-pathology/range-of-entrywounds-in-suicides.html. Available at: https://accessemergencymedicine. $m$ hmedical.com/content.aspx?bookId=351\&sectionId=396197 19 
Molona, D.K and DiMaio V.J (2013) Rifle Wounds: A Review of Range and Location as Pertaining to Manner of Death. DOI: 10.1097/PAF.0b013e31827ab4b1

Molina, D K, Wood, L and DiMaio, V. J.M.(2007) Shotgun Wounds: A Review of Range and Location as Pertaining to Manner of Death, The American Journal of Forensic Medicine and Pathology: June 2007 - Volume 28 - Issue 2 - p 99-102. doi:

10.1097/01.paf.0000257415.82728.d7

Mussen, D (2020) Firearms (Handguns and rifles). Available at https://www.mussenhealth.us/gunshot-wounds/chapter-6.html

Peonim, V, Srisont, Udnoon, J, Wongwichai S, Thapon A and Worasuwannarak, W (2016) Entrance and exit wounds of high velocity bullet: An autopsy analysis in the event of dispersing the mass rally in Bangkok Thailand, Legal Medicine, volume 23: 10-16. Available at: https://www.sciencedirect.com/science/article/abs/pii/S1344622316300840?via\%3Dihub DOI: 10.1016/j.legalmed.2016.08.010

Pricher,P, Prei, D, Emberger, A.T, Predekmp, M.G, Gesenberger, D (2016) The influence of the bullet shape on the width of abrasion collars and the size of gunshot entrance holes. International Journal of Legal Medicine. https://doi.org/10.1007/s00414-016-1501-6

Randall, B. and Jaqua, R (1990) Gunshot Entrance Wound Abrasion Ring Width as a Function of Projectile Diameter and Velocity, Journal of Forensic Sciences, Vol. 36, No. 1, 0, pp. 138144, https://doi.org/10.1520/JFS13014J. ISSN 0022-1198 
Saukko, P and Knight, B Knight`s Forensic Pathology. $3^{\text {rd }}$ edn. London. Edward Arnold (Ltd). 2015.

Wong Y.S, Khairul O, Atiah AAG, Aziz A, Hilmi B, Hazfalinda N, Albakari SP (2013) Porcine Model: Differences in Entry and Exit Wounds by Semi-Automatic Pistol at Different Shooting Distances, Available at:

https://www.researchgate.net/publication/260060250_Porcine_model_Differences_in_entry_and _exit_wounds_by_semi-automatic_pistol_at_different_shooting_distances

Yong Y.E. A systematic review on ricochet gunshot injuries. Science Direct. 2017; 26: pp. 4551. https://doi.org/10.1016/j.legalmed.2017.03.003 